\title{
S'impliquer dans des groupes de codéveloppement professionnel accompagné : témoignage d'un observateur
}

\author{
Par Vincent Gagnon, UQAC
}

Résumé

Cet article propose une réflexion au sujet de l'implication en recherche à travers un témoignage sur le dispositif méthodologique du groupe de codéveloppement professionnel accompagné. L'originalité de l'article tient au fait qu'il est rédigé du point de vue d'un assistant de recherche qui a été présent à de très nombreux moments de la collecte, à titre de soutien technique. Ayant été un témoin privilégié et ayant entendu la richesse des nombreux échanges, le regard porté sera donc transversal et intégrateur. Le point de vue partagé a le potentiel de rejoindre une catégorie du lectorat curieux de mieux comprendre l'intérêt de s'impliquer en recherche, par un angle original différent de celui du chercheur. Dans un article de type scientifique, un chercheur tente généralement d'expliquer le contexte de sa recherche, sa méthodologie, ses résultats et la portée de ceux-ci. Dans le texte proposé, il s'agit d'un article à caractère professionnel où la posture est celle d'un assistant de recherche qui a participé uniquement à la collecte de données, sans intervenir activement lors de celle-ci. L'article abordera les avantages, les inconvénients et les retombées potentielles du groupe de codéveloppement professionnel accompagné et plus globalement de l'implication en recherche pour un enseignant.

Mots-clés: implication en recherche, groupe de codéveloppement professionnel accompagné, assistant de recherche, enseignant, enseignant associé, stagiaire, formation, formateur. 


\section{REVUE HYBRIDE DE L'ÉDUCATION}

\section{Introduction}

Avec le Consortium régional de recherche en éducation (CRRE) en activité au Saguenay-Lac-St-Jean depuis bientôt 20 ans, il est commun de nos jours de rencontrer dans un établissement scolaire des enseignants et des enseignantes s'impliquant au sein de projets de recherche. Cela n'est pas surprenant puisque les chercheurs en éducation proposent de plus en plus de recherches participatives aux acteurs du terrain. Lorsque de telles recherches sont mises en place, on retrouve généralement d'une part, un chercheur ou une équipe de recherche et d'autre part, les acteurs du milieu tels que les enseignants, les conseillers pédagogiques, les directions d'établissement ou les autres intervenants scolaires. Au sein de l'équipe de recherche, on oublie parfois de mentionner les assistants de recherche qui participent aux rencontres et sont présents à différents moments du projet. Les assistants de recherche sont généralement des étudiants aux cycles supérieurs qui sont passionnés par la recherche et saisissent les opportunités de vivre des expériences en recherche auprès de chercheurs chevronnés. Bien qu'ils fassent partie de l'équipe de recherche, leur degré d'implication est variable selon le rôle et les tâches qui leur sont attribués. Ainsi, leur contribution peut aller, par exemple, d'une participation active aux réflexions de l'équipe de recherche, à la création d'outils de collecte, au traitement et à l'analyse des données, ou encore à une simple présence en soutien technique lors des interactions entre chercheurs et enseignants. Je suis doctorant en éducation et lors d'un projet de recherche ayant eu lieu de 2009 à 2012, j'ai occupé un rôle d'assistant de recherche où mes tâches consistaient principalement en des tâches plus techniques telles que s'occuper des enregistrements audio et de la prise de notes. Le projet de recherche visait à renforcer le savoir d'expérience, les compétences et la professionnalité des formateurs de la relève enseignante en milieu de pratique. II avait comme objectifs d'investiguer le savoir d'expérience des enseignants associés, de systématiser ce savoir à des fins de diffusion, de le bonifier avec des pairs et des 


\section{REVUE HYBRIDE DE L'ÉDUCATION}

membres de l'équipe de recherche et d'accélérer le développement de l'agir compétent par le partage et la mise en valeur de ce savoir au sein de la communauté de pratique constituée dans le cadre du projet. Ce projet regroupait des enseignants associés du primaire et du secondaire répartis dans différentes écoles rattachées à l'une ou l'autre des quatre commissions scolaires de la région du Saguenay-Lac-St-Jean. En dépit d'une contribution modeste à cette recherche, cette opportunité m'a donné la chance et le privilège d'écouter activement les échanges d'un grand nombre d'enseignants et d'observer la dynamique unique qui a pris forme au sein des différents groupes de codéveloppement auxquels je prenais part. N'ayant pas vraiment eu la chance de m'exprimer sur ce que j'ai vu et entendu, puisque je n'étais pas impliqué dans le traitement des données ni dans les communications liées au projet, cet article vise à partager mes réflexions en tant qu'observateur d'un dispositif méthodologique que je qualifie de fort intéressant et d'enrichissant pour les participants. II s'agit d'une initiative personnelle visant à mieux faire connaitre ce qu'est le groupe de codéveloppement professionnel accompagné et surtout, à discuter de l'intérêt de s'impliquer en recherche. L'intention derrière cet article est avant tout de démystifier ce que peut représenter l'implication dans un projet de recherche collaborative pour un enseignant n'ayant jamais vécu d'expérience en ce sens, à travers le regard d'un observateur privilégié. Dans cet article, j'aborderai tout d'abord en quoi consiste le dispositif du groupe de codéveloppement professionnel accompagné, je poursuivrai avec les avantages, les inconvénients, et les retombées potentielles de l'implication en recherche.

\section{Le groupe de codéveloppement professionnel accompagné}

Cette section cherche à illustrer concrètement une des formes que peut prendre un projet de recherche pour un participant qui a fait le choix de s'impliquer. II existe différents dispositifs méthodologiques. Le dispositif retenu par le projet consistait au groupe de codéveloppement professionnel accompagné. Tout d'abord, il faut savoir qu'un groupe de codéveloppement professionnel est considéré comme une « approche de 


\section{REVUE HYBRIDE DE L'ÉDUCATION}

formation qui mise sur le groupe et sur les interactions entre les participants pour favoriser l'atteinte de l'objectif fondamental : améliorer la pratique professionnelle» (Payette et Champagne, 1997, p.7). La dimension accompagnée de cette approche de formation provient d'une adaptation originale faite au modèle de Payette et Champagne (1997) par l'équipe de recherche qui a mené le projet. Cette nouvelle formule, en plus du modèle de base, intègre un chercheur universitaire qui accompagne le groupe et peut intervenir tant sur le contenu que le processus dans les groupes de codéveloppement professionnel.

Les différents groupes de codéveloppement professionnel auxquels j'ai pris part étaient composés d'environ une dizaine d'enseignants associés qui se réunissaient à quelques reprises pendant leur année scolaire pour réaliser une étude de cas. Le déroulement des séances se faisait en six étapes où chaque groupe étudiait une situation professionnelle différente vécue avec un stagiaire et présentée par un participant du groupe aux collègues présents. Par exemple, une enseignante associée pouvait partager une problématique en lien avec l'attitude condescendante de sa stagiaire, ou des problèmes récurrents de mauvaise planification. II n'y avait pas de thème ou de sujet prédéfinis, mais d'une séance à l'autre, l'équipe essayait de proposer des situations différentes. Avant chaque séance, l'équipe de recherche se concertait pour sélectionner une situation professionnelle qui serait discutée au sein du groupe. Bien qu'un chercheur universitaire était présent à chaque rencontre, les échanges étaient structurés et animés par un enseignant membre de l'équipe de recherche. Voici un survol rapide des six étapes de la démarche.

Étape 1: Une expérience vécue par un enseignant en lien avec sa fonction d'enseignant associé est présentée. Pendant l'exposé de la situation, le groupe écoute sans poser de questions. L'analyse du cas est faite collectivement lors des étapes suivantes. 


\section{REVUE HYBRIDE DE L'ÉDUCATION}

Étape 2 : Les participants interrogent la personne qui a présenté la situation dans le but d'obtenir plus d'informations et de précisions en lien avec certains aspects du cas présenté. À cette étape, les participants ne proposent pas de solutions, ils tentent seulement de clarifier des éléments du contexte de la situation.

Étape 3 : L'enseignant synthétise la situation présentée en une phrase ou deux et exprime ensuite ce qu'il attend du groupe à travers le travail collectif. Les attentes peuvent être variées et personnelles, allant d'une analyse de la situation pour mieux comprendre la situation vécue, à une recherche de solutions afin d'être mieux outillé si une situation semblable devait se présenter à nouveau.

Étape 4: Les participants partagent leur analyse de la situation, expriment leur point de vue, donnent leur avis et font des suggestions. Le tout s'effectue dans une perspective constructive où chacun met à contribution ses savoirs pratiques et ses connaissances théoriques afin d'apporter un éclairage collectif. Le chercheur universitaire intervient au besoin pour apporter, par exemple, un éclairage issu des connaissances scientifiques, et inversement, les participants peuvent l'interroger pour obtenir des informations complémentaires. Lors de cette étape, l'enseignant associé qui a présenté la situation écoute et se retient de prendre la parole.

Étape 5 : La personne ayant présenté le cas résume les éléments qu'elle considère les plus signifiants et présente ce qu'elle envisage faire. Autrement dit, elle mentionne ce qu'elle retient ou a appris plus particulièrement parmi tout ce qui a été exprimé par les membres du groupe. Elle précise aussi comment elle aborderait une pareille situation dans l'avenir.

Étape 6 : Les participants prennent quelques minutes pour écrire ce qu'ils retiennent de la rencontre afin de noter les apprentissages réalisés. Un tour de table a lieu où ils sont invités à exprimer leurs idées sur ce qu'ils ont appris et ce qu'ils retiennent de la situation explorée. 


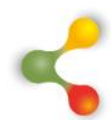

\section{REVUE HYBRIDE DE L'ÉDUCATION}

Une fois la dernière étape complétée, le groupe prend le temps d'évaluer le déroulement de la rencontre afin d'en réguler le fonctionnement, au besoin. Une rencontre de codéveloppement professionnel permet de traiter d'un cas en profondeur et se déroule sur une période d'environ 90 minutes.

\section{Les avantages de s'impliquer dans un projet de recherche}

Un bon nombre d'enseignants ne cherchent pas nécessairement d'opportunités de rejoindre un projet de recherche. Cela peut se comprendre, leur tâche est vaste et complexe: les planifications, la correction, la gestion au quotidien, les nombreuses tâches courantes, l'encadrement d'un stagiaire pour certains, etc. Considérant l'ampleur du défi logistique que représentent les tâches de l'enseignant, il ne serait donc pas approprié d'ajouter la culpabilité de ne pas prendre part à un projet de recherche. Malgré le fait qu'il y aura toujours des raisons pouvant être invoquées pour décliner une invitation à joindre un projet de recherche, les avantages de s'impliquer sont réels et méritent d'être abordés.

Dans le cadre de mon implication comme assistant de recherche avec les groupes de codéveloppement professionnel, j'ai eu la chance d'identifier quelques-uns de ces avantages qui ont suscité mon intérêt. Tout d'abord, lorsque des enseignants se joignent à un tel projet, ils ne se connaissent pas tous a priori. Se retrouver dans un groupe composé en tout ou en partie d'inconnus peut créer une petite gêne au début, mais la passion pour l'enseignement qui habite les participants prend rapidement le dessus. J'ai ainsi pu observer des groupes où les participants étaient parfois timides à s'exprimer lors des premières rencontres, mais la dynamique a rapidement évolué vers un esprit de communauté de pratique où la confiance régnait et un véritable sentiment d'appartenance s'est développé. Les participants se retrouvent alors dans un contexte leur permettant d'échanger collectivement à partir d'expériences individuelles, où leurs connaissances et leur créativité à trouver des 


\section{REVUE HYBRIDE DE L'ÉDUCATION}

solutions sont mises en valeur. Dans le projet des groupes de codéveloppement professionnel, cela s'est notamment traduit par l'enrichissement du répertoire de stratégies et de moyens pour mieux accompagner les stagiaires. Les problèmes partagés et les solutions avancées et discutées par le groupe ont mené à développer l'expertise et les compétences professionnelles des enseignants présents et, ce faisant, renforcer leur identité en tant que formateurs de la relève enseignante.

Les avantages présentés ci-dessus touchent avant tout à la dimension professionnelle, mais d'autres avantages tacites peuvent aussi être mentionnés. Par exemple, lors des rencontres, j'ai pu observer qu'un climat convivial et respectueux prévalait lors des échanges. Un tel climat contribue positivement à l'expérience et fait en sorte que les participants reviennent d'une rencontre à l'autre. De plus, certains groupes prennent un temps pour planifier des petites bouchées ou encore des rafraichissements. J'ai aussi eu la chance de prendre part à quelques rencontres de codéveloppement au restaurant. Bien évidemment, il faut s'impliquer pour les bonnes raisons, mais cela illustre bien que le dispositif comporte une série d'étapes; le cadre, lui, est souple et flexible et permet de vivre une expérience enrichissante au plan interpersonnel.

\section{Les obstacles et les craintes liées à l'implication dans un groupe de codéveloppement}

J'ai hésité à mettre cette section dans l'article, mais à des fins de transparence et pour mettre en évidence les deux côtés de la médaille, j'ai cru bon de la conserver. Cela dit, de mon point de vue d'observateur, il m'est apparu que s'impliquer présentait plus d'avantages que d'inconvénients. Puisque cet article s'adresse notamment à des enseignants n'ayant pas vécu d'expérience d'implication au sein d'un projet de recherche, cette section pourra leur apparaitre particulièrement intéressante. 


\section{REVUE HYBRIDE DE L'ÉDUCATION}

Tout d'abord, il faut le reconnaitre, s'impliquer dans un groupe de recherche demande pour certains de sortir de leur zone de confort. J'entends par là qu'il est parfois plus simple de conserver sa routine, puisque la routine est généralement rassurante. Par contre, en allant de l'avant et en devenant participant dans un projet de recherche, on franchit un pas qui nous ouvre sur des possibilités fort intéressantes. En s'impliquant, on risque notamment de faire de nouveaux apprentissages qui pourront s'intégrer dans ses pratiques, ou encore de faire découvrir des pratiques inspirantes à ses collègues. Les occasions d'échanger et de discuter de ses compétences professionnelles ou de son savoir-faire enseignant sont parfois rares selon les milieux. Bien que sortir de sa zone de confort puisse représenter une crainte ou un désagrément pour certains, ce qui ressort de mes observations sur le terrain m'amène à constater qu'on y gagne beaucoup plus en allant de l'avant et en participant activement à un projet de recherche.

Deuxièmement, en prenant la décision de s'impliquer, on accepte d'introduire d'autres personnes dans son cercle, on s'ouvre et on se confie à eux. En pareille situation, il peut y avoir une certaine peur du jugement d'autrui, une peur d'apparaitre vulnérable ou de faire remettre ses pratiques en question. II est vrai que s'impliquer demande de l'ouverture, mais comme je l'ai mentionné précédemment, le climat qui s'installe lors des rencontres en est un de respect et d'écoute. J'ai pu voir, lors de certaines rencontres, des participants qui ont présenté des cas invraisemblables et complexes. Des cas où la tournure des évènements a provoqué la fin d'un stage en cours, et où il aurait été facile de juger, car la solution peut parfois apparaitre évidente aux yeux des autres avec le recul. Cependant, il est généralement plus facile d'entrevoir une solution à un problème alors qu'on est extérieur à celui-ci, et aussi parce que chacun a un parcours unique qui l'a outillé différemment. Demeurer isolé, se conforter dans ses certitudes et oublier de se remettre en question est une recette qui peut nous mener à répéter les mêmes erreurs. L'humilité et le courage dont font preuve les participants en entrant en dialogue 


\section{REVUE HYBRIDE DE L'ÉDUCATION}

avec leurs collègues sont exemplaires et jamais je n'ai vu ni entendu de propos disant le contraire. Au final, les participants qui ont exposé la situation problématique qu'ils rencontraient en ressortent avec une liste de solutions à essayer et surtout, plus confiants, plus outillés, et moins isolés.

\section{Les retombées de s'impliquer dans un projet de recherche}

Après avoir discuté de quelques avantages et obstacles ou craintes liés à l'implication dans un groupe de codéveloppement comme participant, j'aborde maintenant les retombées potentielles qui peuvent en découler. Ces retombées sont multiples et touchent différentes sphères à des niveaux autant individuels que collectifs, mais aussi à l'échelle de la classe, de l'école, des commissions scolaires et des milieux de formation eux-mêmes.

Tout d'abord, pour le participant qui s'implique dans un projet, tel que celui des groupes de codéveloppement professionnel accompagné, les retombées au plan personnel et professionnel sont riches. Par exemple, le bagage de stratégies et de solutions qu'un participant retire lors des rencontres lui permet d'intervenir différemment auprès des stagiaires qu'il a sous sa supervision. De plus, les récits et échanges au sujet des cas vécus par ses collègues lui permettent d'éviter de possibles problèmes en anticipant les situations avant qu'elles ne surviennent, et d'être mieux préparé à faire face à celles qui se présentent à lui. C'est le cas notamment d'un groupe auquel je participais où un enseignant associé avait une routine d'accueil des stagiaires particulièrement efficace pour clarifier les attentes réciproques. Les participants de ce groupe s'en sont inspirés et l'ont réutilisée lors de l'accueil de leurs nouveaux stagiaires. Qui plus est, j'ai souvenir du témoignage d'une participante qui se faisait de plus en plus solliciter dans son milieu et qui prenait plaisir à répondre aux questions de ses collègues. Elle avait aussi mentionné avoir senti qu'elle était devenue une référence et en était très fière. Cette

situation est arrivée dans le cadre du projet des groupes de 


\section{$<$}

\section{REVUE HYBRIDE DE L'ÉDUCATION}

codéveloppement, mais peut aussi survenir dans bien d'autres recherches où les enseignants sont invités à participer. En outre, la présence d'un chercheur universitaire lors des rencontres permet au participant de créer ou renouer un lien de communication et de confiance avec l'université. Ce lien permet aux participants de prendre conscience du rôle des enseignants dans l'amélioration de la formation initiale et l'encadrement des stagiaires, en plus de faciliter le transfert des connaissances issues de la recherche.

Deuxièmement, pour le milieu scolaire lui-même, un enseignant qui a participé à un groupe de recherche devient un acteur clé au sein de son équipe-école. En effet, les collègues qui n'ont pas participé peuvent bénéficier de l'expertise développée par le participant. Ainsi, un enseignant associé qui a participé au projet en lien avec l'encadrement des stagiaires devient un agent de rayonnement des connaissances issues de la recherche participative à laquelle il a pris part. II peut ainsi communiquer et partager à ses collègues les apprentissages et savoirs acquis dans son groupe de codéveloppement mais aussi dans ceux des autres groupes puisqu'il est invité à participer aux rencontres élargies organisées par le CRRE où sont mis en commun les résultats issus du projet de recherche. Considérant les efforts et ressources investis par les commissions scolaires et les écoles pour la formation continue, avoir en ses murs des enseignants qui s'impliquent en recherche est clairement un atout considérable.

Troisièmement, une retombée importante de l'implication des enseignants associés au projet décrit dans cet article consiste en l'amélioration, l'actualisation et la bonification de la formation des formateurs de stagiaires. Cela fut le cas pour le présent projet, mais ça l'est tout autant pour divers projets de recherche. L'enseignement et les approches de formation doivent continuer d'évoluer pour suivre les développements et les transformations de notre société. II est primordial de mettre l'accent sur l'importance de s'impliquer en recherche pour contribuer à permettre au système d'éducation de demeurer en 


\section{REVUE HYBRIDE DE L'ÉDUCATION}

adéquation avec l'évolution des connaissances du domaine. Les chercheurs ne sont pas là pour dicter aux praticiens quoi faire, et pour parvenir à dispenser une formation universitaire de qualité qui prépare adéquatement les futurs enseignants, une synergie entre praticiens et chercheurs doit exister. Les acteurs du monde de l'éducation sont des gens créatifs qui innovent pour amener les élèves à apprendre. Autant les praticiens que les chercheurs ont la capacité d'innover et c'est notamment par l'implication en recherche que peuvent converger leur créativité respective. Pour faire état des innovations développées sur le terrain, il est impératif que les chercheurs viennent observer, expérimenter, documenter et décrire ce qui s'y passe, et cela ne peut se faire sans impliquer les praticiens. Par conséquent, l'une des retombées fondamentales de l'implication en recherche pour un participant à un projet de recherche consiste en l'amélioration de la formation des futurs maitres.

\section{Conclusion}

Tout comme dans les milieux hospitaliers où la population souhaite que leurs médecins soient au fait des plus récents traitements et découvertes, les parents et les élèves sont en droit de s'attendre à ce que le personnel du milieu de l'éducation soit au fait des plus récentes avancées en termes de connaissances. Pour y parvenir, le monde de la recherche en éducation ne peut recourir à des microscopes, il a besoin de la collaboration du personnel enseignant pour mener des études qui permettent de prendre le pouls de l'innovation du terrain, d'expérimenter des approches nouvelles, et de mettre en lumière les pratiques prometteuses. Qu'il s'agisse de pratiques d'accompagnement des stagiaires ou de toute autre question liée à l'éducation, l'implication en recherche est nécessaire et sera toujours la bienvenue. En terminant, il est important de retenir qu'en plus d'être une voie importante d'actualisation des connaissances, l'implication en recherche représente aussi un mécanisme essentiel de transfert des connaissances issues de la recherche. Si cet article a suscité chez vous la curiosité et le désir de 


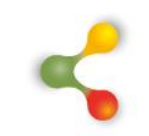

\section{REVUE HYBRIDE DE L'ÉDUCATION}

vivre une expérience d'implication sur un projet de recherche, vous êtes invités à communiquer auprès de votre direction d'école et à demeurer à l'affut des offres qui sont diffusées dans les milieux.

\section{Références}

Payette, A. et Champagne, C. (1997). Le groupe de codéveloppement professionnel. Sainte-Foy, QC : Presses de I'Université du Québec. 